\title{
Electronic Properties of Isolated Nickel in Diamond
}

\author{
R. Larico ${ }^{\dagger}$, J. F. Justo ${ }^{\ddagger}$, W. V. M. Machado ${ }^{\dagger}$, and L. V. C. Assali ${ }^{\dagger}$, \\ ${ }^{\dagger}$ Instituto de Física, Universidade de São Paulo, CP 66318, 05315-970, São Paulo, SP, Brazil \\ ${ }^{\ddagger}$ Escola Politécnica, Universidade de São Paulo, CP 61548, CEP 05424-970, São Paulo, SP, Brazil
}

Received on 31 March, 2003

\begin{abstract}
Isolated nickel impurities in diamond have been investigated using the spin-polarized full-potential linearized augmented plane wave total energy method. The electronic and atomic structures, symmetries, transition energies, and formation energies of substitutional and interstitial $\mathrm{Ni}$ impurities in diamond were computed. The results were discussed in the context of the electrically active centers in synthetic diamond.
\end{abstract}

\section{Introduction}

Synthetic diamond is generally grown by high pressure-high temperature methods using transition metals alloys (containing manganese, iron, nickel, and cobalt) as catalysts. Nickel, which is incorporated into the resulting diamond crystal, has been unambiguously related to a large number of electrically and optically active centers. Electron paramagnetic resonance (EPR) and optical absorption measurements have shown that these centers are either related to isolated $\mathrm{Ni}$ $[1,2,3]$ or to Ni-dopant complexes [4]. Some absorption lines are stronger under high nitrogen concentrations, while others are stronger under low nitrogen concentrations. Although some nickel-nitrogen complexes could be formed [4], nitrogen will only shift the Fermi level of the system, activating or deactivating a certain isolated nickel center[5].

As a result of intensive experimental investigations, a microscopic model has been built for the interactions of $\mathrm{Ni}$ with the diamond crystalline lattice. Nickel has a $3 \mathrm{~d}^{8} 4 \mathrm{~s}^{2}$ atomic valence configuration, which will hybridize with nearest-neighboring carbon atoms in the host crystal. Interstitial nickel was identified in the positive charge state $\left(\mathrm{Ni}_{i}^{+}\right)$ with a spin $1 / 2$ in trigonal or tetrahedral symmetry $[2,3]$. Substitutional nickel was identified in the negative charge state $\left(\mathrm{Ni}_{s}^{-}\right)$with a tetrahedral symmetry and a spin 3/2 [1].

Previous theoretical investigations of $\mathrm{Ni}$ in diamond has been restricted to calculations using small cluster models $[6,7]$, in which atomic relaxations have not been considered. However, as it will be shown latter, relaxations play a major role on the electronic properties of those Ni-related defects. Here, we used the full-potential linearized augmented plane wave (FP-LAPW) method [8, 9], implemented into the WIEN97 package [10], to investigate the electronic and structural properties of nickel-related defects in diamond. Our results are discussed in the context of the experimentally identified electrically active centers in synthetic diamond.

\section{Methodology}

We used total energy methods to investigate the substitutional and interstitial $\mathrm{Ni}$ impurities in diamond. The calculations were performed within the density functional theory [11], combined with the Perdew-Burke-Ernzerhof exchange-correlation potential [12]. In the FP-LAPW framework, two distinct regions are defined inside the simulation cell: the spherical atomic regions and an interstitial region. In the later, the electronic wave-functions were expanded in a plane-wave basis set, with a cutoff depending on the choice of the atomic sphere radii. For bulk diamond, a convergence in the total energy was achieved by using 7.0/R (maximum length of the plane-waves), where $\mathrm{R}$ is the smallest radius of the spheres defining the host atoms. We used small atomic radii $\left(\mathrm{R}_{\mathrm{C}}=\mathrm{R}_{\mathrm{Ni}}=1.2\right.$ a.u. $)$, as opposed to larger atomic radii with touching spheres $(R=1.46$ a.u. in the equilibrium lattice constant). This choice would allow large relaxations on the internal degrees of freedom without any atomic sphere overlap. In the atomic regions, we used a spherical harmonic basis set with azimuthal quantum number up to $\ell=10$ and a non-spherical contribution up to $\ell_{n s}=4$. The carbon $1 \mathrm{~s}$ and the nickel $1 \mathrm{~s}, 2 \mathrm{~s}$, and $2 \mathrm{p}$ atomic wavefunctions were treated as core states. The Brillouin zone (BZ) was sampled by a $6 \times 6 \times 6$ grid of $k$-points [13], which is reduced to $16 k$-points in the irreducible BZ (IBZ) for the primitive cell. Self-consistent interactions were performed until convergence on both the total energy $\left(10^{-4} \mathrm{eV}\right.$ per unit cell) and total charge in the atomic spheres $\left(10^{-5}\right.$ electronic charges per atom) was achieved. Table I presents the theoretical results of crystalline diamond compared to the experimental data [14].

The electronic structure of Ni impurities in diamond was studied by considering a 54-atom reference supercell and a $2 \times 2 \times 2$ grid [13], corresponding to a unique point to integrate in the IBZ. The convergence criteria were the same as the ones described earlier. The atomic positions were relaxed until the forces were smaller than $0.05 \mathrm{eV} / \AA$.

Table I: Theoretical structural and electronic properties of diamond: lattice constant $(a)$, bulk modulus (B), energy band gap $\left(\mathrm{E}_{\mathrm{g}}\right)$, and cohesive energy $\left(\mathrm{E}_{\mathrm{coh}}\right)$.

\begin{tabular}{lcccc}
\hline & $a(\AA)$ & $\mathrm{B}(\mathrm{Mbar})$ & $\mathrm{E}_{\mathrm{g}}(\mathrm{eV})$ & $\mathrm{E}_{\mathrm{coh}}(\mathrm{eV})$ \\
\hline this work & 3.570 & 4.31 & 4.12 & -7.73 \\
expt. [14] & 3.567 & 4.42 & 5.48 & -7.37 \\
\hline
\end{tabular}




\section{Isolated Ni defects}

Figure 1 displays the impurity energy levels for the tetrahedral interstitial $\mathrm{Ni}$ (in neutral and positive charge states) and the substitutional $\mathrm{Ni}$ (in neutral and negative charge states) centers around the $\Gamma$ point.

The neutral interstitial nickel center in diamond $\left(\mathrm{Ni}_{\mathrm{i}}^{0}\right)$, shown in fig. 1(a), presents a closed shell structure with a fully occupied e level in the band gap and has a $T_{d}$ symmetry. The $\mathrm{T}_{\mathrm{d}}$ crystal field splitting of $1.8 \mathrm{eV}$ splits the Ni $3 \mathrm{~d}-$ related energy levels into a $t_{2}$ plus an e level, the former being resonant in the valence band. This behavior is similar to the neutral interstitial Ni impurity in silicon [15]. The nickel nearest-neighbor and next-nearest-neighbor atoms undergo outward relaxations of $0.28 \AA$ and $0.11 \AA$, respectively. This center in a positive charge state $\left(\mathrm{Ni}_{\mathrm{i}}^{+}\right)$and in a $\mathrm{T}_{\mathrm{d}}$ point symmetry, shown in fig 1(b), has an effective spin of $1 / 2$. For the positive charge state, the crystal field splitting is essentially the same as the one for the neutral charge state. On the other hand, the exchange splitting $\left(\Delta \mathrm{E}_{\mathrm{xc}}\right)$ acts mostly over the gap energy level with e symmetry, being about $0.15 \mathrm{eV}$. This effect drives the center to a low spin configuration. The electronic structure of the $\mathrm{Ni}_{i}^{+}$center is fully consistent with the microscopic model proposed to explain the EPR signals of synthetic diamond grown from a Ni-solvent with the addition of a nitrogen getter [2].

The neutral substitutional nickel center in a $T_{d}$ point symmetry $\left(\mathrm{Ni}_{\mathrm{s}}^{0}\right)$ in diamond, shown in fig. 1(c), presents an effective spin $S=1$. The $T_{d}$ crystal field of $3.2 \mathrm{eV}$ splits the $\mathrm{Ni} 3 \mathrm{~d}$-related energy levels into an e plus a $\mathrm{t}_{2}$ level, the former being resonant in the valence band. The exchange splitting acts over all levels, being $\Delta \mathrm{E}_{\mathrm{xc}}(\mathrm{e})=0.3 \mathrm{eV}$ and $\Delta \mathrm{E}_{\mathrm{xc}}\left(\mathrm{t}_{2}\right)=0.6 \mathrm{eV}$, driving the center to a low spin configuration. The configuration of $\mathrm{Ni}_{\mathrm{s}}^{0}$ in diamond is similar to the theoretical result of the same center in silicon, which also gives an effective spin $S=1$ [15]. The nickel nearestneighbor atoms undergo an outward relaxation of $0.23 \AA$. This center presents small distortions which are not shown in the figure.

The substitutional nickel center in negative charge state $\left(\mathrm{Ni}_{\mathrm{s}}^{-}\right)$, shown in fig. $1(\mathrm{~d})$, has a $\mathrm{T}_{\mathrm{d}}$ point symmetry and presents an effective spin $S=3 / 2$. The $T_{d}$ crystal field of 3.7 $\mathrm{eV}$ splits the $\mathrm{Ni} 3 \mathrm{~d}$-related energy levels into an e plus $\mathrm{a}_{2}$ level, the former being resonant in the valence band. The exchange splitting acts over all levels, being $\Delta \mathrm{E}_{\mathrm{xc}}(\mathrm{e})=0.5 \mathrm{eV}$ and $\Delta \mathrm{E}_{\mathrm{xc}}\left(\mathrm{t}_{2}\right)=0.9 \mathrm{eV}$, driving the center to a low spin configuration. The nickel nearest-neighbor atoms undergo an outward relaxation of $0.23 \AA$, very similar to the relaxation for this center in the neutral charge state.

\section{Formation and transition energies}

The formation energy of a Ni impurity in a q charge state, $\left(\Delta \mathrm{E}_{\mathrm{f}}{ }^{\mathrm{q}}\right)$ is given by

$$
\Delta \mathrm{E}_{\mathrm{f}}^{\mathrm{q}}=\mathrm{E}\left(\mathrm{n}_{\mathrm{C}}, \mathrm{n}_{\mathrm{Ni}}, \mathrm{q}\right)-\mathrm{n}_{\mathrm{C}} \mu_{\mathrm{C}}-\mathrm{n}_{\mathrm{Ni}} \mu_{\mathrm{Ni}}+\mathrm{q}\left(\mu_{\mathrm{e}}+\varepsilon_{\mathrm{v}}\right),
$$

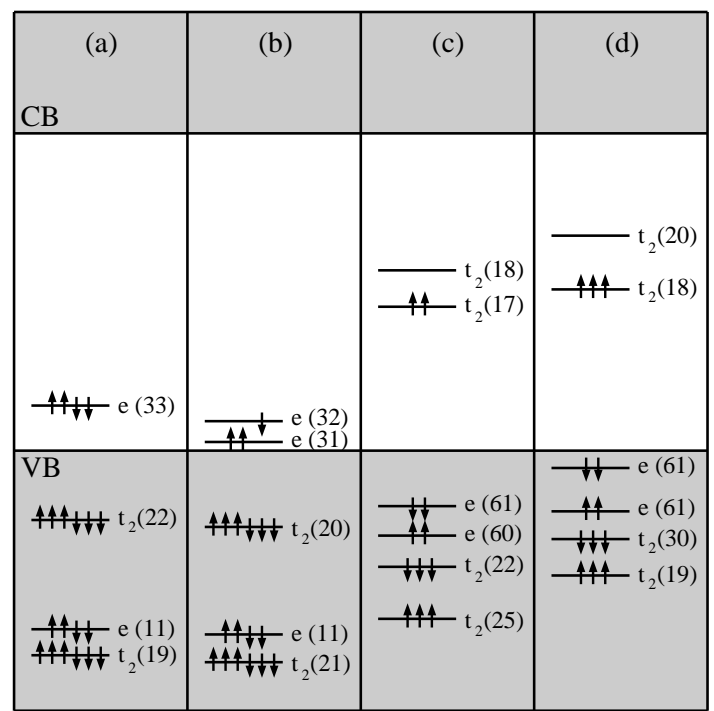

Fig. 1. The Kohn-Sham energy eigenvalues representing the $\mathrm{Ni}$ 3d-related impurity levels near the gap region: (a) $\mathrm{Ni}_{\mathrm{i}}^{0}$, (b) $\mathrm{Ni}_{\mathrm{i}}^{+}$, (c) $\mathrm{Ni}_{\mathrm{s}}^{0}$, and (d) $\mathrm{Ni}_{\mathrm{s}}^{-}$centers. The level occupation is given by the number of $\uparrow$ (spin up) and $\downarrow$ (spin down) arrows, respectively. The percentage of $\mathrm{Ni} 3 \mathrm{~d}$-character of the energy levels inside the atomic spheres are represented by the numbers in parenthesis. The figure only displays those energy levels which are related to the Ni impurity, as computed by the charge percentage with $\mathrm{d}$ character. VB and $\mathrm{CB}$ symbols represent the valence and conduction bands.

where $\mathrm{E}\left(\mathrm{n}_{\mathrm{C}}, \mathrm{n}_{\mathrm{Ni}}, \mathrm{q}\right)$ is the total energy of a supercell calculation with $n_{\mathrm{C}}$ carbon atoms and $\mathrm{n}_{\mathrm{Ni}} \mathrm{Ni}$ atoms, and $\mu_{\mathrm{C}}$ and $\mu_{\mathrm{Ni}}$ are the chemical potentials for $\mathrm{C}$ in a diamond structure and $\mathrm{Ni}$ in a fcc structure, respectively. The electron chemical potential $\mu_{\mathrm{e}}$ defines the Fermi level position in the band gap (with respect to the top of the valence band, $\varepsilon_{\mathrm{v}}$ ).

The computed formation energy $\left(\Delta \mathrm{E}_{\mathrm{f}}\right)$ of the interstitial nickel, in neutral charge state, is $8.7 \mathrm{eV}$ higher than the energy of the substitutional nickel in the same charge state. Based only in formation energy considerations, substitutional nickel is considerably more favorable than interstitial nickel. Using the total energies of the centers in positive and negative charge states, we computed the transition energies. The acceptor transition energy (-/0) of the $\mathrm{Ni}_{\mathrm{S}}$ is $\varepsilon_{\mathrm{v}}+2.9 \mathrm{eV}$, which is in excellent agreement with the experimental data of $\varepsilon_{\mathrm{v}}+3.0 \mathrm{eV}[16,17]$. The donor transition energy $(0 /+)$ of the $\mathrm{Ni}_{\mathrm{i}}$ is computed to be $\varepsilon_{\mathrm{v}}+0.5 \mathrm{eV}$.

\section{Summary}

In summary, we performed an investigation on the electronic and structural properties of isolated nickel impurities in diamond. For most of the nickel centers studied here, our results are fully consistent with proposed models based on the EPR and optical experiments on synthetically grown diamond. Additionally, the computed acceptor transition energy for the $\mathrm{Ni}_{\mathrm{s}}$ center is in agreement with experimental data. 


\section{Acknowledgments}

This work was supported by Brazilian agencies FAPESP and CNPq. The calculations were performed at the LCCACCE of the Universidade de São Paulo.

\section{References}

[1] J. Isoya, H. Kanda, J. R. Norris, J. Tang, and M. K. Bowman, Phys. Rev. B 41, 3905 (1990).

[2] J. Isoya, H. Kanda, Y. Uchida, Phys. Rev. B 42, 9843 (1990).

[3] M. H. Nazaré, A. J. Neves, and G. Davies, Phys. Rev. B 43, 14196 (1991).

[4] S. C. Lawson and H. Kanda, J. Appl. Phys. 73, 3967 (1993).

[5] G. Davies, Phyica B 273-274, 15 (1999).

[6] Y. Jinlong, Z. Manhong, and W. Kelin, Phys. Rev B 49, 15525 (1994).

[7] J. Goss, A. Resende, R. Jones, S. Öberg, and P. R. Briddon, Mater. Sci. Forum 196-201, 67 (1995).

[8] P. Blaha, K. Schwarz, and P. Sorantin, Comput. Phys. Commun. 59, 399 (1990).
[9] D. J. Singh, Planewaves, Pseudopotentials and the LAPW method(Kluver Academic, Norwell, 1994).

[10] P. Blaha, K. Schwarz, and J. Luitz, WIEN97, A Full Potential Linearized Augmented Plane Wave Package for Calculating Crystal Properties (Karlheinz Schwarz, Techn. Universitatat Wien, Austria), 1999.

[11] P. Hohenberg and W. Kohn, Phys. Rev. 136, B864 (1964).

[12] J. P. Perdew, S. Burke, and M. Ernzerhof, Phys. Rev. Lett. 77, 3865 (1996).

[13] H. J. Monkhorst and J. D. Pack, Phys. Rev. B 13, 5188 (1976).

[14] Landolt-Börnstein, Numerial Data and Functional Relationships in Science and Technology, vol. 17, edited by $\mathrm{O}$. Madelung, M. Schulz, and H. Weiss (Springer-Verlag, New York, 1982).

[15] F. Beeler, O. K. Andersen, and M. Scheffler, Phys. Rev. B 41, 1603 (1990).

[16] D. M. Hofmann, P. Christmann, D. Volm, K. Pressel, L. Pereira, L. Santos, and E. Pereira, Mater. Sci. Forum 196201, 79 (1995).

[17] D. M. Hofmann, M. Ludwig, P. Christmann, D. Volm, B. K. Meyer, L. Pereira, L. Santos, and E. Pereira, Phys. Rev. B 50, 17618 (1994). 